\title{
Research on the Cognitive Style and Course Satisfaction of Students Majoring in Art and Design
}

\author{
Guo Zhiqiang, Liu Meijun* \\ Email address: \\ 984951487@qq.com (Guo Zhiqiang),meijun0728@126.com (Liu Meijun) \\ ${ }^{*}$ Corresponding author
}

Academy of Art and Design, Inner Mongolia University of Science and Technology, Baotou, China

To cite this article:

Guo Zhiqiang, Liu Meijun. Research on the Cognitive Style and Course Satisfaction of Students Majoring in Art and Design. Science Innovation. Vol. 7, No. 1, 2019, pp. 43-47. doi: 10.11648/j.si.20190701.19

Received: March 21, 2019; Accepted: May 7, 2019; Published: May 23, 2019

\begin{abstract}
With more and more attention paid to higher education by our country, college students' cognition behavior and characteristics have become an important basis for the reform of higher education and teaching. The teaching methods should be carried out according to the students of different majors. Especially in the context of diversified information, college students' cognitive style, learning habits and value judgments have formed rapidly and have an important impact on them. There are some differences in behavior preference and thinking mode between students majoring in art and design with others. Based on a sample survey of students majoring in art and design in Colleges and universities, this paper studies their learning habits, cognitive characteristics, course satisfaction and demands, and respects students' professional and group differences, and provides a certain research basis for constructing the expected classroom form and putting forward more suitable teaching methods.
\end{abstract}

Keywords: Art and Design, Cognitive Style, Course Satisfaction, Educational Research

\section{艺术设计专业学生认知方式与课程满意度研究}

郭志强, 刘美君 ${ }^{*}$

内蒙古科技大学艺术与设计学院, 包头, 中国

邮箱

984951487@qq.com（郭志强）, meijun0728@126.com（刘美君）

摘要：随着国家对高等教育的关注和重视, 大学生认知、行为及特点成为高校教育教学改革的重要依据, 课堂教学方 法需根据不同专业的学生针对性开展, 尤其在信息多元化的背景下, 大学生的认知方式、学习习惯及价值判断快速形 成并产生重要影响, 其中, 艺术设计专业的学生行为偏好和思维方式与其他专业学生存在一定的差异, 本文通过对高 校艺术设计专业学生进行抽样调查, 研究学生在学习环境下的学习习惯、认知特点、课堂满意度及课堂诉求, 尊重学 生的专业差异和群体差异, 为构建期望的课堂形式、提出更适用的教学方式提供一定的研究基础。

关键词: 艺术设计, 认知方式, 课程满意度, 教学研究 


\section{1. 研究基础}

\section{1. 理论基础}

本文基于Herman A. Witkin和J. P. Guilford的认知方 式理论展开研究, 认知方式具备个体差异性, 同时又具有 群体共同性, 是人们在处理认识活动和信息加工时所传递 出来的固定风格, 能够让人们产生习惯化的行为模式, 尤 其是Witkin的认知方式理论在教育行业中有强有力的实 践依据, 场依存型和场独立型、沉思型和冲动型、辐合型 和发散型这三个维度的认知方式是本文认知方式研究的 主要内容。[1]场依存型表现为容易关注周围环境信息并对 其产生影响, 愿意接受他人的建议; 场独立型不易受外部 因素的干扰, 受内部线索的影响较多, 能够独立做出判断 和决定, 有自己的想法和思考。沉思型表现为形成思维的 过程较慢, 习惯于全面考虑问题之后再做出具体结论, 且 结论的准确性较高; 冲动型表现为快速做出反应, 并急于 将问题表达出来, 通常更加强调快速解决问题, 而忽略结 论的精准度。辐合型认知指在解决问题时先搜集相关信息, 然后运用逻辑规律将问题范围逐渐缩小, 直至找到解决问 题的办法; 发散型表现为思维方式的分类和扩散, 想法不 局限于某一具体方面, 最后提出的解决办法通常不仅限于 一种, 而是多个不同的方向。[2]基于以上三个维度, 这六
类认知方式各有其优势和弊端, 没有好坏之分, 每个人的 认识方式都存在差异性。

\section{2. 研究现状}

美国认知教育心理学家布鲁纳提出了认知结构论, 即 学生的认知学习观和教师的结构教学观; 奥苏伯尔根据学 习的方式和学习材料与学生原有知识结构的关系提出了 有意义接受学习论; 加涅认为学习的模式是用来说明学习 结构与过程的, 并提出了信息加工学习论。目前我国认知 心理学的研究已较为成熟, 并将认知研究应用于高校教学 以提高课程质量和满意度, 本文对艺术设计专业的学生进 行抽样调查, 旨在将认知领域的研究用于艺术设计专业的 教育教学改革中。

\section{3. 研究背景}

采用问卷调查的方式, 对内蒙古科技大学艺术设计专 业学生进行学习习惯、认知方式及课堂满意度研究, 共收 回有效问卷169份, 对调研结果进行统计分析并进行频数 分析、分类汇总、交叉分析以及相关关系分析, 定性总结 学生的学习习惯、行为以及认知, 并定量分析学生对于艺 术设计类课程授课形式的态度和期望。

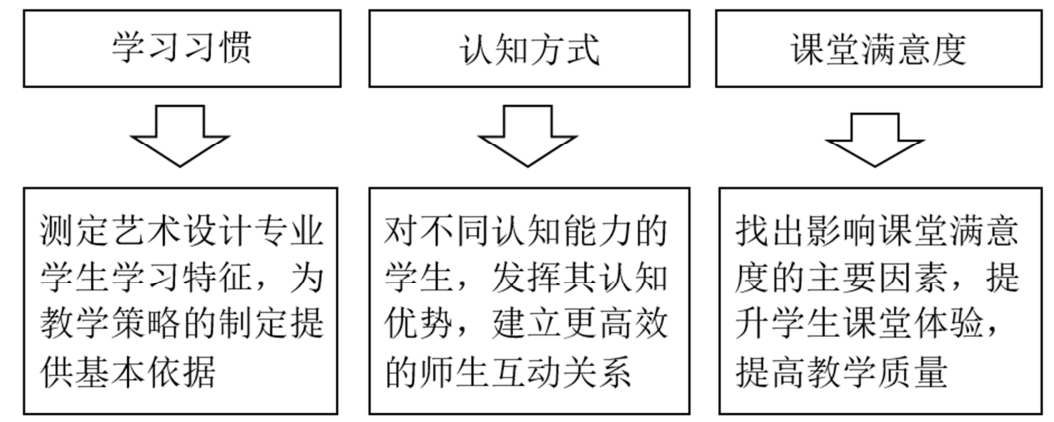

图1 艺术设计专业学生学习影响因素研究策略。

\section{2. 调研结果与分析}

\section{1. 学生学习习惯的影响因素调查分析}

学生学习习惯主要受内部因素和外部因素的影响, 其 中内部因素主要包含行为层次和意识层次, 表1统计结果 的基数表明, 总体上艺术设计专业学生学习容易受外部环 境因素的影响, 对于学习场所具有一定的要求, 善于制定 学习计划, 但在计划实施方面存在一定欠缺, 相比外部行 为层的表现, 在深层次的学习认知和学习耐力上需要进一 步自我强化。但是, 学习习惯因学习成绩和年级的不同而 呈现出一定的变化趋势, 从成绩角度分析, 相对优秀的学 生受外部环境因素的影响较大, 有固定的学习习惯, 能够 根据自身特点制定计划并实施，而成绩较差的学生 $36 \%$ 没 有固定的学习习惯, 并且没有明确的学习方向和目的。从 年级高低来看, 通常刚入学的大学生由于对新环境有新鲜 感, 并且仍保留着高等教育以前的学习习惯, 有较好的自 我约束力, 随着年级的增长, 学生逐渐出现学习惰性, 尤
其在意识层面逐渐放松, 大三年级出现低峰值, 在学习习 惯、学习意识和行为上达到最低点, 但大四年级的学生由 于毕业和就业压力, 面临学习困境时善于提出问题并积极 寻求解决办法, 整体的学习意识出现回升的趋势。

\section{2. 学生认知方式的影响因素分析}

研究艺术与设计专业学生的认知方式类别, 并分析性 别、成绩和专业是否对认知规律有一定的影响。据研究表 明, 场依存型的学生倾向于教育学、心理学等文科学科, 场独立型的学生更多选择建筑、自然科学、数学等理工科 学科, 认知方式的差异产生了一定程度的专业分化, 并且 不同认知方式的学生通常会选择与个体认知相匹配的学 科领域, 这一点验证了男生多数选择理工科, 女生大多选 择文科的现象。[3]从图2中可以看出, 男生的认知方式中 场独立型占多数, 且学习成绩相对优秀的学生多数属于场 独立型认知方式, 场依存和场独立这两类认知方式的学生 适用于不同的教学策略, 场独立型学生能够发挥主观能动 性, 对于抽象的概念能够快速接受, 场依存型学生相对来 
说比较被动, 当面对具体的概念时学习效果比较有优势, 因此成绩和认知方式并不是简单的线性关系, 教学材料的 呈现形式、课程内容等都对不同认知方式的学生产生不同 的效果。[4]但是在互联网信息多样化和碎片化的今天, 大 学生认知方式呈现出统一的变化趋势, 由场独立型向场依
存型转变, 同时学生能够快速高效的获取多种渠道的知识, 这在一定程度上降低了学生对教师的依附性, 而场依附型 认知方式的学生较易受环境信息的影响, 教师应引导学生 积极的价值取向, 学生应加强对知识的篮选和判断能力, 吸收有价值的文化信息。

表1 艺术设计专业学生学习习惯和成绩/年级的相关关系。

\begin{tabular}{|c|c|c|c|c|c|c|c|c|c|}
\hline \multirow[b]{2}{*}{$\mathbf{X} \backslash \mathbf{Y}$} & \multicolumn{3}{|c|}{ 表层学习策略 } & \multicolumn{3}{|c|}{ 深层学习认知 } & \multicolumn{2}{|l|}{ 外部因素 } & \multirow[b]{2}{*}{$\begin{array}{l}\text { 没有固定地 } \\
\text { 学习习惯 }\end{array}$} \\
\hline & $\begin{array}{l}\text { 习惯自学, } \\
\text { 制定个人 } \\
\text { 学习计划 }\end{array}$ & $\begin{array}{l}\text { 运用课余时 } \\
\text { 间, 合理预习 } \\
\text { 和复习 }\end{array}$ & $\begin{array}{l}\text { 高效利用 } \\
\text { 课堂时间 }\end{array}$ & $\begin{array}{l}\text { 能够长时 } \\
\text { 间集中精 } \\
\text { 力 }\end{array}$ & $\begin{array}{l}\text { 独立钻研, } \\
\text { 善于思考 }\end{array}$ & $\begin{array}{l}\text { 有意识地 } \\
\text { 训练思维 } \\
\text { 和独创性 }\end{array}$ & $\begin{array}{l}\text { 重视学习 } \\
\text { 场所 }\end{array}$ & $\begin{array}{l}\text { 遇到问题会 } \\
\text { 及时向外界 } \\
\text { 提问 }\end{array}$ & \\
\hline 大一/优秀 & $83.33 \%$ & $83.33 \%$ & $100 \%$ & $100 \%$ & $66.67 \%$ & $83.33 \%$ & $83.33 \%$ & $83.33 \%$ & $16.67 \%$ \\
\hline 大一/良好 & $41.67 \%$ & $37.5 \%$ & $62.5 \%$ & $37.5 \%$ & $54.17 \%$ & $50 \%$ & $79.17 \%$ & $45.83 \%$ & $12.5 \%$ \\
\hline 大一/中等 & $33.33 \%$ & $22.22 \%$ & $33.33 \%$ & $27.78 \%$ & $22.22 \%$ & $22.22 \%$ & $61.11 \%$ & $22.22 \%$ & $16.67 \%$ \\
\hline 大一/及格 & $25 \%$ & $25 \%$ & $25 \%$ & $25 \%$ & $25 \%$ & $25 \%$ & $75 \%$ & $25 \%$ & $50 \%$ \\
\hline 大二/优秀 & $83.33 \%$ & $50 \%$ & $50 \%$ & $66.67 \%$ & $50 \%$ & $66.67 \%$ & $83.33 \%$ & $50 \%$ & $16.67 \%$ \\
\hline 大二/良好 & $36 \%$ & $20 \%$ & $40 \%$ & $24 \%$ & $36 \%$ & $36 \%$ & $44 \%$ & $36 \%$ & $40 \%$ \\
\hline 大二/中等 & $38.10 \%$ & $38.10 \%$ & $19.05 \%$ & $19.05 \%$ & $38.10 \%$ & $33.33 \%$ & $52.38 \%$ & $23.81 \%$ & $33.33 \%$ \\
\hline 大二/及格 & $20 \%$ & $0.00 \%$ & $40 \%$ & $0.00 \%$ & $40 \%$ & $0.00 \%$ & $20 \%$ & $40 \%$ & $40 \%$ \\
\hline 大三/优秀 & $33.33 \%$ & $33.33 \%$ & $66.67 \%$ & $33.33 \%$ & $66.67 \%$ & $66.67 \%$ & $100 \%$ & $66.67 \%$ & $0.00 \%$ \\
\hline 大三/良好 & $60 \%$ & $13.33 \%$ & $20 \%$ & $53.33 \%$ & $26.67 \%$ & $6.67 \%$ & $73.33 \%$ & $26.67 \%$ & $13.33 \%$ \\
\hline 大三/中等 & $33.33 \%$ & $26.67 \%$ & $40 \%$ & $6.67 \%$ & $20 \%$ & $20 \%$ & $53.33 \%$ & $26.67 \%$ & $20 \%$ \\
\hline 大三/及格 & $100 \%$ & $50 \%$ & $50 \%$ & $50 \%$ & $50 \%$ & $50 \%$ & $100 \%$ & $50 \%$ & $0.00 \%$ \\
\hline 大四/良好 & $44.44 \%$ & $22.22 \%$ & $22.22 \%$ & $33.33 \%$ & $33.33 \%$ & $33.33 \%$ & $44.44 \%$ & $33.33 \%$ & $11.11 \%$ \\
\hline 大四/中等 & $38.46 \%$ & $23.08 \%$ & $30.77 \%$ & $15.38 \%$ & $30.77 \%$ & $38.46 \%$ & $53.85 \%$ & $38.46 \%$ & $46.15 \%$ \\
\hline 大四/及格 & $15.38 \%$ & $7.69 \%$ & $30.77 \%$ & $23.08 \%$ & $23.08 \%$ & $7.69 \%$ & $46.15 \%$ & $30.77 \%$ & $23.08 \%$ \\
\hline
\end{tabular}

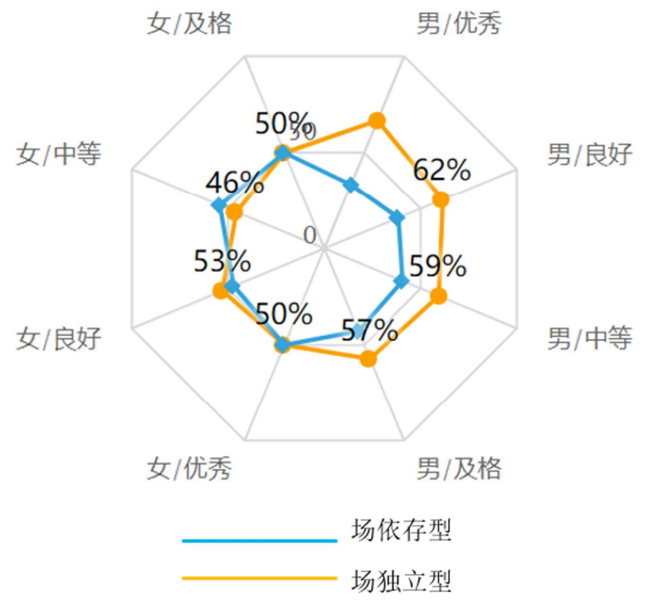

图2 场依存型和场独立型认知方式。

从图3中得出结论, 从性别角度来看, 在艺术设计专业 学生中, 男生中辐合思维的比例明显高于女生, 从成绩角度 分析, 优秀的学生中辐合型思维的人数较多, 随着成绩的变 化, 成绩一般的学生中发散型思维的人数越来越多。辐合和 发散是相互协调的, 在艺术设计领域进行创新创造的过程中, 其产物不能局限于有限的逻辑思维中, 因此学生既需要有发 散思维, 还需要有辐合思维。在发散阶段通过头脑风暴提出 新的尝试和创意, 而后又能够及时将思维收玫, 避免过度发 散、难于收玫, 导致设计想法不切实际。同样, 也要避免学 生在设计初期就过于收玫, 难以产生新创意, 导致设计过程 遇到瓶颈。因此, 当面临两种类型的学生时, 教师需用不同 的方式引导学生, 例如鼓励男生培养发散型思维, 鼓励女生 锻炼辐合型思维, 以达到在设计流程中两种思维方式的平衡。
沉思型和冲动型的认知方式更多的体现在信息处理、 思考及形成结论时的速度和准确度, [5]从图4中能够看出, 整体上男生中沉思型的数量多于女生, 学习成绩较优秀的 学生中沉思型数量明显高于冲动型, 而成绩一般的学生中 沉思型和冲动型的差异逐渐减小并趋于接近。一般来说, 冲动型学生往往粗心大意, 表现出一定程度的学习能力缺 失, 学习成绩相对较差, 但是, 在涉及到多任务的学习活 动中时, 冲动型学生则能发挥其认知优势, 较好的完成目 标。[6]沉思型学生则能够克服其结论滞后的现状, 往往比 较有耐力, 能够适当约束自己的行为, 更加关注结论是否 全面具体, 是否更符合逻辑。教师在面临冲动型学生时, 需要帮助学生克服认知缺失, 培养学生掌握分析问题的方 法, 并通过语言、视觉的引导, 帮助其克服冲动本能。

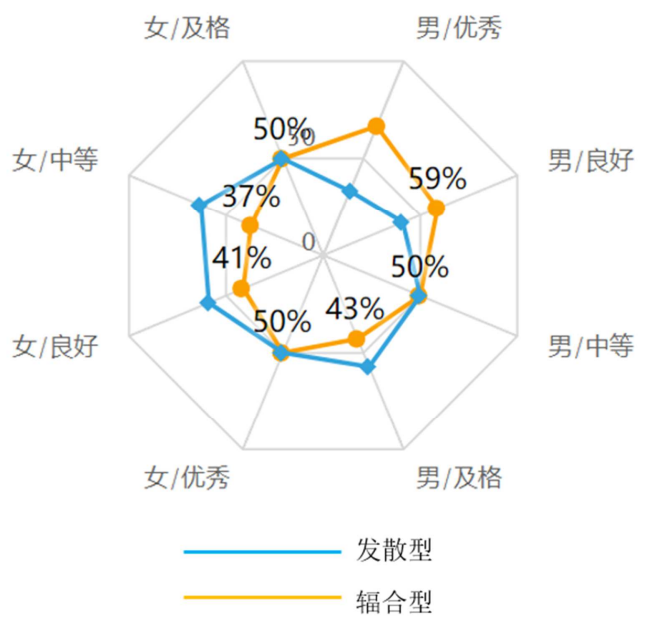

图3 辐合型和发散型认知方式。 


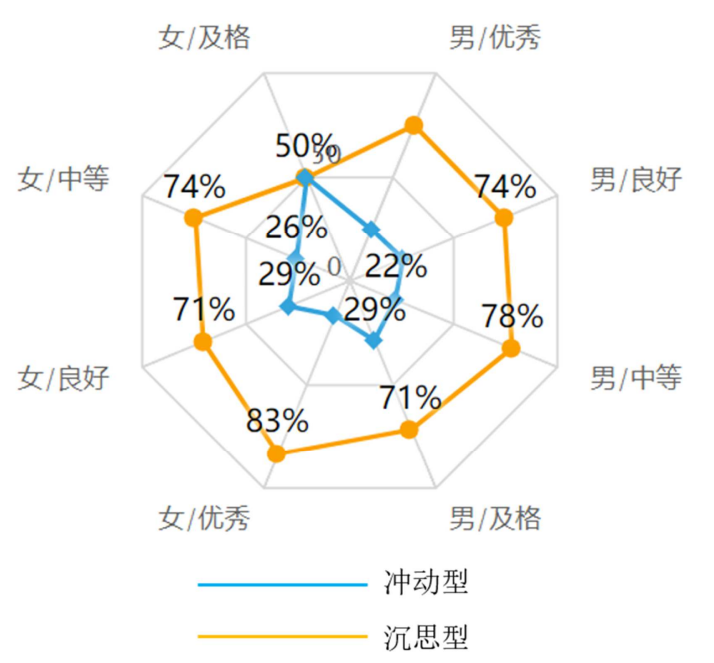

图4 冲动型和沉思型认知方式。

\section{3. 学生对认知能力的诉求分析}

认知能力通常指大脑对信息的加工处理能力, 对学生 而言, 学生获取知识和应用知识的能力总体归结于记忆力、 感觉、知觉、思维和想象力, 表 2 是对艺术设计专业学生在 专业课程中的认知能力诉求, 想象力被 $88 \%$ 的学生认为是 最重要的能力, 其次是思维、记忆力、感觉、知觉, 艺术 设计专业的特点不仅在于学科知识的获取, 还有对交叉学 科知识的综合运用能力, 以及知识转化设计的过程, 而在 知识转化时, 仅仅记忆力、理解和知觉的能力调动是不够 的, 对学生的思维模式、联想及想象的能力提出了更高的 要求。因此从表中数据可以看出, 学生对思维和想象力的 期望值较高, 尤其是成绩一般的学生对于想象力的诉求更 加强烈, 从侧面反映出学生在学习实践中, 在发挥联想思 维和想象力的过程中遇到了瓶颈, 同时认为这两项能力是 发挥专业特长的重要指标, 针对这一想象, 教师需要加强 创意训练和意识培养, 启迪和帮助学生掌握创新思维方式。
表2 艺术设计专业学生对认知能力的诉求。

\begin{tabular}{|c|c|c|c|c|c|}
\hline $\mathbf{X} \backslash \mathbf{Y}$ & 记忆力 & 感觉 & 知觉 & 思维 & 想象力 \\
\hline 优秀 & $66.67 \%$ & $38.89 \%$ & $33.33 \%$ & $77.78 \%$ & $83.33 \%$ \\
\hline 良好 & $52.05 \%$ & $58.90 \%$ & $34.25 \%$ & $80.82 \%$ & $90.41 \%$ \\
\hline 中等 & $53.73 \%$ & $44.78 \%$ & $28.36 \%$ & $82.09 \%$ & $85.07 \%$ \\
\hline 及格 & $45.45 \%$ & $54.55 \%$ & $18.18 \%$ & $72.73 \%$ & $90.91 \%$ \\
\hline
\end{tabular}

\section{4. 学生课堂满意度调查分析}

学生对于课堂的满意程度对学生学习和接受知识的程 度产生重要的影响, 虽然满意度的调查结果因学生个体标准 不同存在一定的偏差, 但“教与学”是学生和教师相互影响的 活动, 学生的满意与否成为各高校衡量教学质量的重要指标, 高校纷纷引进教学评价系统, 通过信息化网络和大数据分析 对教师、学生、学校三方评价构建教学评价体系。[7]表3中, 从艺术设计专业学生对于当前课堂满意度调查中发现了一 些问题, 例如学生对教学环境与设备的满意度普遍偏低, 并 且期望能够更新和创新教学模式, 采用多样化的授课方式组 织课堂活动。将满意度调查和学生年级进行交叉分析时发现, 低年级的学生对于课堂的评价大部分集中在教学环境与设 施方面, 高年级的学生则更重视教学模式 (如表4), 关注 的重点由外部环境转移到学习活动本身, 高年级的学生对教 师的授课内容、教学组织形式提出了更高要求。

表3 艺术设计专业学生课堂满意度调查。

\begin{tabular}{lll}
\hline 选项 & 比例 \\
\hline 不喜欢所学专业 & & $8.33 \%$ \\
教学模式守旧不创新 & & $50 \%$ \\
教师授课方式枯燥单一 & $38.89 \%$ \\
所处的教学环境不佳 & & $55.56 \%$ \\
教学设备陈旧 & & $69.44 \%$ \\
\hline
\end{tabular}

表4 艺术设计专业学生课程满意度 (年级) 调查。

\begin{tabular}{llllll}
\hline $\mathbf{X} \backslash \mathbf{Y}$ & 不喜欢所学专业 & 教师的授课方式枯燥 & 所处的教学环境不佳 & 教学设备陈旧 & 教学模式守旧不创新 \\
\hline 大一 & $0.00 \%$ & $36.36 \%$ & $72.73 \%$ & $81.82 \%$ & $45.45 \%$ \\
大二 & $0.00 \%$ & $30 \%$ & $40 \%$ & $100 \%$ & $30 \%$ \\
大三 & $9.09 \%$ & $54.55 \%$ & $45.45 \%$ & $36.36 \%$ & $72.73 \%$ \\
大四 & $50 \%$ & $25 \%$ & $75 \%$ & $50 \%$ & $50 \%$ \\
\hline
\end{tabular}

将不同专业背景的学生及其课堂期望进行交叉分析, 发现文科背景的学生更期望体验新型课堂形式, 例如虚拟 现实课堂、翻转课堂, 理科专业背景的学生对新型课堂的 意愿程度明显低于文科生。虚拟现实课堂体现出较强的沉 浸性和交互性, 若将其应用于艺术设计专业课程构建中,
学生能够对产品、空间、环境进行交互体验, 这种体验型 课堂突破了原有的教学方式, 在工业产品设计和环境艺术 设计专业教学中模拟三维产品和空间环境, 能够调动听觉、 触觉等多种感官体验, 帮助提高课堂质量、构建趣味课堂 和启发式教学[8]。

表5 不同背景的艺术设计专业学生课堂意愿调查。

\begin{tabular}{llllll}
\hline $\mathbf{X} \backslash \mathbf{Y}$ & 黑板板书 & 多媒体图文讲授课堂 & 沉浸式学习课堂 & 体验式交互课堂 & 翻转课堂 \\
\hline 理科背景 & $25 \%$ & $53.95 \%$ & $25 \%$ & $69.74 \%$ & $38.16 \%$ \\
文科背景 & $3.98 \%$ & $61.29 \%$ & $41.94 \%$ & $70.97 \%$ & $50.54 \%$ \\
\hline
\end{tabular}




\section{3. 启示与结论}

学生认知方式的调查为教学研究提供了理论依据, 目 前, 我国各高校普遍以统一的授课方式实施教学, 这对不 同认知方式的学生产生了不同的学习效果, 势必导致部分 学生知识接受程度较差。一方面, 在教学时可根据不同认 知类型的学生以及专业课程需求交替使用不同教学方式, 例如, 在设计方法课程中, 需要学生通过实地调查发现人 群需求, 需求的提出不能靠学生的主观臆断, 这将对场独 立型的学生提出更高要求, 需要学生听取目标人群的现状 及诉求, 根据人群特点判断需求, 避免陷入个人的思维局 限中。在创意设计课程中, 前期需要学生掌握头脑风暴的 能力, 辐合型认知方式更擅长沿着特定方向进行逻辑推理, 而发散型认知的学生则能从不同的方向发散思维并输出 不同的结论。[9]在创意课程后期, 头脑风暴之后需要提出 一个最佳方向进行深入研究, 在深入研究阶段辐合型认知 的学生则能够更好的发挥认知优势, 将设计方案完善到更 加具体充实。因此, 针对不同的学习内容, 各认知风格的 学生都能够匹配适用的教学方式, 需要教师针对学生特点 具体分析, 分类匹配与失配, 让认知方式不同的学生在各 教学环节中都能够找到自己的位置, 锻炼学习能力, 提升 学习效果。[10]另外, 多媒体及新技术的发展为构建新型 课堂提供了更可靠的支持, 尤其是VR课堂的高度沉浸和 交互性颠覆了传统的教学方式, 学生也对这一课堂方式表 现出较强烈的意愿, 同时VR技术能够对艺术设计专业学 生的设计成果更好的进行可行性验证与分析, 这将是设计 类专业教学方式的重要突破。

\section{致谢}

本文为内蒙古自治区高校创新创业教育科学研究课 题《虚拟现实技术在艺术设计学科教育中的应用》
(NMSC18084) 和内蒙古科技大学教育教学改革研究项 目《基于认知科学及用户体验的高校教学策略再构建》 （JY2018057）的阶段性成果之一。

\section{参考文献}

[1] 段伟丽.认知风格发展研究[J].文教资料,2012(23):37-38。

[2] 程涛,张茂林.认知方式理论的整合与发展 [J].济南大学学报 (社会科学版),2004(02):78-82。

[3] 王远思. 思想政治教育视野下的理工科高校校园文化建设 [D].武汉工程大学, 2012。

[4] 庞翠菊.场依存性认知方式理论及其应用研究 [J]. 学理 论,2013(17):48-49。

[5] 李倩.浅析认知风格差异对合作学习的影响及其对策[J]. 黑 龙江史志,2008(Z1):123-124+96。

[6] 祝茵.学习风格的个别差异与教学对策设计 [J]. 昆明理工大 学学报(社会科学版),2002(03):56-59。

[7] 王运武,杨曼.从高校学生课堂教学满意度透视课堂教学创 新性变革[]].现代远程教育研究,2016(06):65-73。

[8] 章新成.虚拟现实技术在工业设计专业人才培养中的应用 研究[J].美术教育研究,2015(24):179。

[9] 杨翼. 艺术设计类学生图形创意思维模式养成训练研究 - 以跨学科移植思维训练为例 [J]. 美与时代 (中),2014(04):53-54。

[10] 张鸿. 关注学生学习风格差异的课堂教学反思 $[\mathrm{D}]$.四川师 范大学, 2005 。 